\title{
Surgical Resection of Thoracic Aortic Aneurysms in Wiskott-Aldrich Syndrome
}

\author{
Mehmet Akif Onalan, MD,${ }^{1}$ Omer Ali Sayin, $\mathrm{MD},{ }^{2}$ Emin Tireli, $\mathrm{MD}^{2}$ \\ ${ }^{1}$ Department of Cardiovascular Surgery, Sanliurfa Mehmet Akif Inan Training and Research Hospital, Sanliurfa, Turkey; ${ }^{2}$ Department \\ of Cardiovascular Surgery, Istanbul University Istanbul Medical Faculty, Istanbul, Turkey
}

\section{ABSTRACT}

Aortic aneurysms are a rare condition in children. Wiskott-Aldrich syndrome is a primary immunodeficiency characterized by infections, thrombocytopenia, and eczema. Aortitis and aneurysm formation seem to be progressive in patients with this condition. The risk of death from aneurysmal rupture in patients with Wiskott-Aldrich syndrome is high, and surgery is required for the resection of these aneurysms. We report a case wherein a successful resection of a descending thoracic aneurysm was performed. A 12 year-old child with this syndrome underwent a one-stage descending aortic aneurysm repair under continuous visceral perfusion. Histological examination showed the presence of an aortitis with granulomatous inflammatory response and multinucleated cells.

\section{INTRODUCTION}

Wiskott-Aldrich syndrome is a rare $\mathrm{X}$-linked disorder characterized by eczema, thrombocytopenia and immunodeficiency. Early death is common as a result of infection, bleeding and malignancy [Perry 1980]. Rare cases of vasculitis and aneurysm formation have been reported in Wiskott-Aldrich syndrome, but the association has not been well identified [McCluggage 1999]. We report a case of Wiskott-Aldrich syndrome where the patient had progressive aneurysm formation and underwent a successful operation involving the replacement of the descending aorta. The patient remains well and continues to be under regular surveillance.

\section{CASE REPORT}

A 12-year-old child with Wiskott-Aldrich syndrome was followed up in our institution three months ago due to dilatation of the descending aorta. Bone marrow transplantation was considered but could not be carried out, as no matching sibling donor was available. His weight was $26 \mathrm{~kg}$, and his height was $125 \mathrm{~cm}$. The patient had a history of chest

Received fanuary 16, 2018; accepted May 1, 2018.

Correspondence: Omer Ali Saym, Istanbul University Istanbul Medical Faculty, Department of Cardiovascular Surgery, Millet Caddesi, Capa/Fatih, Istanbul, Turkey; tel: +902124142000 - 31897, fax: +902125342232 (e-mail: oasayin@hotmail.com). pain, sickness, dyspnea, and recurrent skin and respiratory infections. His platelet count was only $50,000 / \mu \mathrm{L}$. The thoracic computed tomography angiogram revealed significant aneurysm of the descending aorta. The mid descending aorta measured $4.1 \mathrm{~cm}$ with a fusiform shape, tapering down to the level of the diaphragm where it measured $2 \mathrm{~cm}$. The ascending aorta, aortic arch, and infradiafragmatic aort were not aneurysmal. Echocardiography revealed dilatation of the left atrium due to compression of the pulmonary veins by a dilatated descending thoracic aorta.

A left posterolateral thoracotomy was performed via the fourth rib space to achieve adequate exposure of the lower end of the aneurysm. Cardiopulmonary bypass was carried out with cannulation of the common femoral artery and the common femoral vein. Clamps were used proximal and distal of the supradiaphragmatic aneurysmatic aorta. The aorta was transected proximally just beyond the left subclavian artery, and the proximal end of a $22 \mathrm{~mm}$ gortex graft was anastomosed to it under partial bypass. Finally, the aorta was transected $2 \mathrm{~cm}$ below the lower end of the aneurysm onto normal aorta, and the distal anastomosis was completed.

Only irradiated blood and blood products were used during the intra- and post- operative periods, along with additional prophylactic broad spectrum antibiotics, and a close vigil was kept for any signs of infection. Histological study revealed a destructive, full thickness, chronic aortitis with a patchy inflammatory infiltrate consisting of lymphocytes, plasma cells and eosinophils.

Paraplegia and other possible complications were not seen in the post operative period. The patient was discharged on day 14 and remains well three months post-operatively.

\section{DISCUSSION}

Systemic arthritis and aneurismal dilatation of the aorta have rarely been reported in Wiskott-Aldrich syndrome. To our knowledge, a few adult patients and children have been previously reported on in the literature [Pellier 2011; Ono 2009; Faganello 2008; Bernabeu 2007; Narayan 2004; Johnston 2001]. The pathogenesis of aortic aneurysms remains unclear. Inflammatory aortitis is considered a possible etiology of the aortic lesions. Most patients with Wiskott-Aldrich syndrome die early: in the past, the life span of these patients was quite short, with an expected mean survival of 6.5 years [Johnston 2001]. Since the introduction of the splenectomy and bone marrow transplantation, the mean survival has increased to 25 years. As affected individuals are surviving longer, they appear to be at a higher risk of 
lympho-proliferative diseases and vasculitis [McCluggage 1999]. Vasculitis and aneurysm formation are extremely rare but life threatening complications in Wiskott-Aldrich syndrome. Surgical management of aortic aneurysm in these patients is more complex than in the general population because of their higher propensity to infectious and bleeding complications.

The progressive and destructive nature of the disease may lead to sequential aneurysm formation. Nevertheless, it is possible to safely perform aneurysm resection if appropriate precautions are taken, like the use of irradiated blood products, vigilance with respect to post-operative infection, and the use of appropriate antibiotics, along with close liaison with the immunologists.

The risk of death from aneurysmal rupture in patients with Wiskott-Aldrich syndrome is increased because of thrombocytopenia and impaired platelet function, and it is also possible that aneurysm formation and vasculitis are a more common occurrence in these patients than is reported [McCluggage 1999]. Patients with Wiskott-Aldrich syndrome in the presence of evidence of vasculitis should be investigated for possible aneurysmal dilatation [McCluggage 1999]. The progressive nature of the disease demands long-term surveillance for aneurysm formation in the residual native aorta.

Endovascular stent graft implantation has been performed as a less invasive and safe alternative for type B dissection and aneurysms of the descending thoracic aorta [Lambrechts 2003]. However, this was not utilized in our patient, owing to the involvement of the distal arch and for fear of occlusion of the left subclavian artery.

In conclusion, symptomatic patients or asymptomatic patients with aneurysmal dilatation of the asending aorta, aortic arch, or descending aorta measuring $\geq 6 \mathrm{~cm}$ are advised to operation. Surgical management is likely required in symptomatic patients and can be performed without complications.

\section{REFERENCES}

Bernabeu E, Josa M, Nomdedeu B, et al. 2007. One-step surgical approach of a thoracic aortic aneurysm in Wiskott-Aldrich syndrome. Ann Thorac Surg 83:1537-8.

Faganello G, Hamilton M, Wilde P, et al. 2008. Percutaneous closure of false aneurysms of the aorta in Wiskott-Aldrich syndrome. Eur Heart J 29:6.

Johnston SL, Unsworth DJ, Dwight JF, et al. 2001. Wiskott-Aldrich syndrome, vasculitis and critical aortic dilatation. Acta Paediatr 90:1346-8.

Lambrechts D, Casselman P, Schroeyers P, et al. 2003. Endovascular treatment of the descending thoracic aorta. Eur J Vasc Endovasc Surg 26:437-44

McCluggage WG, Armstrong DJ, Maxwell RJ, et al. 1999. Systemic vasculitis and aneurysm formation in the Wiskott-Aldrich syndrome. J Clin Pathol 52:390-2.

Narayan P, Alwair H, Bryan AJ. 2004. Surgical resection of sequential thoracic aortic aneurysms in Wiskott-Aldrich syndrome. Interact Cardiovasc Thorac Surg 3:346-8.

Ono M, Goerler H, Breymann T. 2009. Aneurysm of the aortic root in the setting of Wiskott-Aldrich syndrome. Cardiol Young 19:212-5.

Pellier I, Dupuis Girod S, Loisel D, et al. 2011. Occurrence of aortic aneurysms in 5 cases of Wiskott-Aldrich syndrome. Pediatrics 127:e498-504.

Perry GS, Spector BD, Schuman LM, et al. 1980. The Wiskott-Aldrich syndrome in the United States and Canada (1892-1979). J Pediatr 97:72-8. 